\title{
Closed orbit correction at synchrotrons for symmetric and near-symmetric lattices
}

\author{
Sajjad Hussain Mirza, ${ }^{1,2, *}$ Rahul Singh, ${ }^{1}$ Peter Forck, ${ }^{1}$ and Harald Klingbeil ${ }^{1,2}$ \\ ${ }^{1}$ GSI Helmholtzzentrum für Schwerionenforschung GmbH, Darmstadt, Germany \\ ${ }^{2}$ Technische Universität Darmstadt, Darmstadt, Germany
}

(Received 22 February 2019; published 23 July 2019)

\begin{abstract}
This contribution compiles the benefits of lattice symmetry for global closed orbit correction, which relies on the decomposition and inversion of the orbit response matrix (ORM). A symmetric arrangement of beam position monitors (BPMs) and correctors results in structured ORMs of circulant or block-circulant type. These structured matrices provide favorable properties in terms of robustness of the ORM inversion against modeling errors due to information compression and a transparent interpretation of analytical vector spaces of BPMs and correctors as well as reduced computational complexity for inversion in the case of larger ORMs. A nearest-circulant approximation is introduced for the extension of these benefits for nearsymmetric lattices. A relation between harmonic orbit correction and singular value decomposition (SVD)based orbit correction is presented. The practical advantages of symmetry exploitation are demonstrated with the help of simulations and experiments in the context of synchrotrons of Facility for Antiproton and Ion Research (FAIR).
\end{abstract}

DOI: 10.1103/PhysRevAccelBeams.22.072804

\section{INTRODUCTION}

The closed orbit correction has been an integral part of the synchrotron and storage ring in light sources as well as in hadron machines for stable beam operations [1-3]. Closed orbit correction methods are typically classified as "local" or "global" given the spatial extent of their correction. Local bumps generated by three to four correctors are utilized for orbit correction in a localized region of a synchrotron, while global correction methods rely on the effect of each corrector throughout the synchrotron. The global effect of a single dipole kick $\theta_{c}$ located at a longitudinal location $s_{1}$ is described by the linear solution of Hill's equation for small perturbations [4,5]:

$z\left(s-s_{1}\right)=\theta_{c} \frac{\sqrt{\beta\left(s_{1}\right) \beta(s)}}{2 \sin \left(\pi Q_{z}\right)} \cos \left[Q_{z} \pi-\left|\mu\left(s_{1}\right)-\mu(s)\right|\right]$,

where $s-s_{1}$ is the longitudinal separation from the source of the dipolar kick and $z$ is the transverse orbit position in either plane (i.e., $z$ is either the horizontal or vertical coordinate in the absence of horizontal-vertical coupling).

\footnotetext{
*Corresponding author. s.h.mirza@gsi.de
}

Published by the American Physical Society under the terms of the Creative Commons Attribution 4.0 International license. Further distribution of this work must maintain attribution to the author(s) and the published article's title, journal citation, and DOI. $\beta$ and $\mu$ denote the lattice beta function and phase advance, respectively, and $Q_{z}$ is the coherent betatron tune in either plane. For the synchrotrons that operate near the transition energy $\left(\gamma_{t}\right)$ or cross it during the ramp, an extra term is added to the right-hand side of Eq. (1) for the horizontal plane in order to incorporate the change in the length of closed orbit as a result of the dipolar kick at the location of nonzero dispersion [6]:

$$
z_{D}\left(s-s_{1}\right)=-\theta_{c} \frac{D\left(s_{1}\right) D(s)}{\left(\frac{1}{\gamma^{2}}-\frac{1}{\gamma_{t}^{2}}\right) C} .
$$

Here, $D$ and $C$ represent the dispersion function and the circumference of the synchrotron, respectively. The storage rings of light sources operate much above the $\gamma_{t}$ [7], making this term irrelevant, but for proton accelerators, although transition crossing is often avoided [8], this term can be significant near a transition.

For a finite number of beam position monitors (BPMs) and correctors, Eq. (1) takes the shape of a matrix referred to as the orbit response matrix (ORM) such that

$$
z=\mathbf{R} \Theta,
$$

where $\Theta$ is the corrector settings vector and $z$ is the beam position vector at the BPM locations. In a nutshell, the main concept of global correction is to calculate the corrector strengths that can counteract the existing dipolar field errors such that the orbit distortion measured with the BPMs is minimized. Historically, four distinct methods have served 
the global orbit correction, which include the sliding bump method [9], Minimisation des Carres des Distoution d'Orbite (MICADO) [10], harmonic correction [11], and singular value decomposition (SVD) [5,12]. A variant of the SVD-type correction referred to as eigenvalue decomposition has also been reported $[13,14]$. The sliding bump method involved forming independent local bumps in order to achieve the required positions at the BPM locations and has been phased out in usage. MICADO, also referred to as orthogonal matching pursuit in the signal processing literature $[15,16]$, was devised to find the most effective correctors to minimize the orbit distortion and has robustness and computational issues. Harmonic correction was the first method to discuss the notion of mode-based correction by means of a decomposition of the perturbed orbit into Fourier harmonics which can be corrected individually. However, the validity and efficacy of this method for nonperiodic lattices was not explored. According to the literature, it seems to have been used only when the correction is intended for a few specific spatial modes of the perturbed orbit, e.g., modes around the coherent tune frequency. SVD is a generalized technique based upon diagonalization and inversion of the matrices and, superseding all the above-mentioned methods, has become the de-facto algorithm for orbit correction. The SVD of a real-valued matrix $\mathbf{R}$ is given as [17]

$$
\mathbf{R}=\mathbf{U S V}^{\mathrm{T}},
$$

where $\mathbf{U}$ and $\mathbf{V}$ are the left and right orthogonal matrices, respectively, and $\mathbf{S}$ is the diagonal matrix whose diagonal entries are called singular values. Like harmonic analysis, SVD also provides the liberty of mode-by-mode orbit correction on top of mode truncation and matrix inversion using a transformation of perturbed orbit vector $z$ and corrector settings vector $\Theta$ into the mode space as [5]

$$
\bar{z}=\mathbf{S} \bar{\Theta},
$$

where $\bar{z}=\mathbf{U}^{\mathrm{T}} z$ and $\bar{\Theta}=\mathbf{V}^{\mathrm{T}} \Theta$ are the BPM and corrector vectors in the transformed mode space, respectively. The solution of Eq. (5) gives the required corrector settings for a given perturbed orbit. SVD also has some limitations, particularly when dealing with uncertainty in the process model, as it is a numerical technique and there is no apparent analytic way of associating uncertainties in the lattice parameters to the singular values [18]. Moreover, a lack of physical interpretation of SVD modes, their mutual phase relationship, and dependence on singular values make the uncertainty modeling complicated $[19,20]$. The interdependence between $\mathbf{U}, \mathbf{S}$, and $\mathbf{V}$ matrices also poses a special challenge for systems where matrices need to be updated during orbit correction, i.e., on the acceleration ramp in synchrotrons [21].
In this paper, we present a one-dimensional discrete Fourier transform (DFT)-based diagonalization and inversion of the ORM for symmetric lattices. The technique is based upon the exploitation of circulant symmetry in the lattice and provides information compression into a diagonal matrix, since the left and right orthogonal matrices are standard Fourier matrices (defined later in the text). As a result, any modeling errors in the ORM affect only the elements of the diagonal matrix, making the matrix inversion robust against uncertainties. The physical interpretation of the analytical Fourier mode space can be used for manipulating the closed orbit, e.g., predicting the closed orbit position at the location of "missing" BPMs and rejection of a dispersion-induced orbit shift during correction. Moreover, for larger ORMs, one can benefit from the reduced computational complexity of the technique. This method serves as the transition between previously discussed harmonic analysis and SVD with an exact equivalence for symmetric lattices. Furthermore, a nearest-circulant extension is discussed for broken symmetries, making most of the ideas discussed for symmetric matrices applicable to those of near-symmetric lattices. The coupling and the term given in Eq. (2) are ignored in the calculation of the ORMs, where the latter does not change the structure of the ORM for symmetric lattices.

The paper is arranged as follows: Section II A uses the example of the vertical plane of SIS18 in order to introduce the circulant symmetry in the ORMs, while the equivalence between SVD and DFT-based decomposition is worked out in Sec. II B. Section II C discusses the possibility of a nearest-circulant approximation of the ORMs for the scenario of broken symmetry using the example of the horizontal plane of SIS18. Section II D covers the blockcirculant symmetry in both transverse planes of SIS100. Section III covers the benefits of exploiting the circulant or block-circulant symmetry in the ORM for practical applications.

\section{SYMMETRY IN RESPONSE MATRIX}

Equations (1) and (3) represent the orbit response matrix and show that it contains one global parameter, i.e., the betatron tune, while two local parameters, the beta function and phase advance, depend solely on the BPM and corrector locations. The global parameter determines the general criterion for the number of BPMs and correctors for an effective orbit correction. In order to ensure that modes up to twice the coherent betatron frequencies can be corrected, at least four BPMs and correctors are required per betatron oscillation. The placement of BPMs and correctors is chosen at locations of higher beta function values in order to enhance the sensitivity of the closed orbit correction. However, during the design, the symmetric arrangement of BPMs and correctors is usually neither given the importance it deserves nor exploited even if it 
exists. The only notable discussion of a symmetric layout for BPMs and correctors was found in Ref. [14] but was limited to finding eigenvalues for eigenvector-based orbit correction. Any discussions towards the matrix inversion, relations to SVD, and application to broken symmetry were not made. These issues will be discussed in this report. In this section, we will discuss two kinds of ORM symmetries which exist in two different synchrotrons of the Facility for Antiproton and Ion Research (FAIR) project, SIS18 and SIS100. We use them as practical examples and to extend our findings to broken symmetries.

\section{A. Circulant symmetry of SIS18 vertical ORM}

SIS18 is a 216.72-m-long synchrotron and has a 12-fold symmetric lattice as shown in Fig. 1. It comprises in the vertical plane one BPM and one corrector each per section placed at the same location. In the horizontal plane, only two correctors violate this symmetry, which will be discussed later in the context of broken symmetry. The symmetric arrangement of BPMs and correctors results in an equal phase advance between adjacent BPMs with the same beta function for all BPMs. The correctors also have a similar arrangement resulting in a circulant ORM, in which each row and column is a cyclic permutation of the previous row and column, respectively. The theory of circulant matrices is well established in the literature [22], and one out of many useful properties of circulant matrices, in the context of ORMs, is their diagonalization and decomposition by the DFT of only one row or column. If $\mathbf{R}_{C}$ is a square circulant matrix of dimension $n$, it can be written as a sum of fundamental cyclic permutation matrices $\pi_{n}^{i}$ (see Appendix A), each of which is convoluted by the corresponding element $r_{i}$ of the first row or column of $\mathbf{R}_{C}$ as

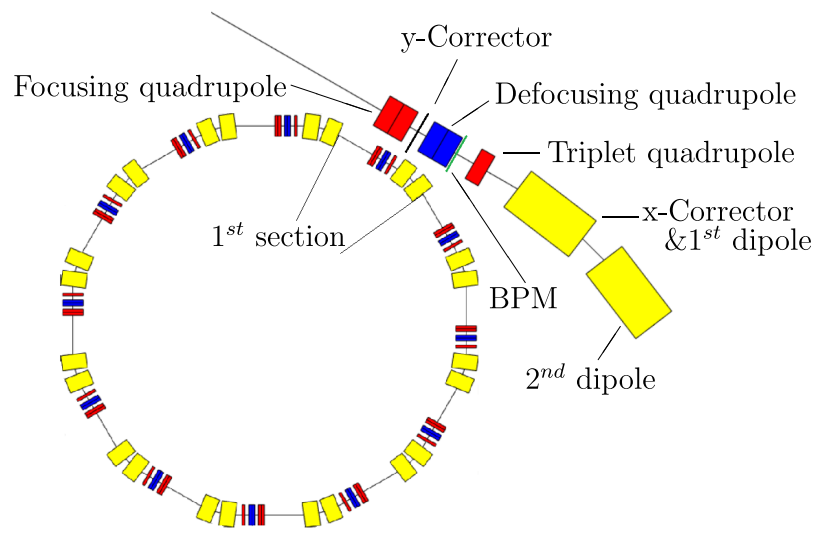

FIG. 1. Schematic of the lattice of the SIS18 synchrotron. The first section has been magnified on the top right, and the components are labeled. The horizontal correctors are located at the first dipole as extra windings in all sections except in the fourth and sixth sections, where they reside on the second dipoles (not labeled in the figure).

$$
\mathbf{R}_{C}=r_{0} \pi_{n}^{0}+r_{1} \pi_{n}^{1}+\cdots r_{n-1} \pi_{n}^{n-1}=\sum_{i=0}^{n-1}\left(\pi_{n}^{i} r_{i}\right),
$$

where $i$ is the order of the permutation matrix. As an example, Eq. (7) shows the SIS18 vertical ORM calculated by MAD-X [23] for triplet optics in units of $\mathrm{mm} / \mathrm{mrad}$ :

$$
\begin{aligned}
\mathbf{R}_{y}= & \operatorname{circ}\left[\begin{array}{lllll}
8.10 & 8.90-8.20-6.40 & 10.2 & 3.30 \\
& -11.20 .1011 .2-3.50-10.16 .60
\end{array} .\right.
\end{aligned}
$$

Here, the operator circ [22] is used to form a matrix by means of cyclic permutations of the row vector it contains as an argument, whose elements are the $r_{i}$ of Eq. (6).

A circulant matrix can be decomposed as

$$
\mathbf{R}_{C}=\mathbf{F}^{*} \Lambda \mathbf{F}
$$

where $\mathbf{F}$ is a standard Fourier matrix which is identical for all circulants of the same size with elements given as [24]

$$
(\mathbf{F})_{f, i}=\frac{1}{\sqrt{n}} e^{j 2 \pi f i / n}=\frac{1}{\sqrt{n}}\left[\cos \left(\frac{2 \pi f i}{n}\right)+j \sin \left(\frac{2 \pi f i}{n}\right)\right]
$$

for $i, f \in[0, \ldots, n-1]$, where $i$ represents the sampling points, $f$ is the discrete frequency of each Fourier mode (column of $\mathbf{F}$ ), $j$ is the imaginary unit, and $n$ is the size of the square circulant matrix. $\boldsymbol{\Lambda}$ is a diagonal matrix containing the discrete Fourier coefficients $\sigma_{f}$ of the first row or column of $\mathbf{R}_{C}$ on its diagonal positions, which are given as

$$
\sigma_{f}=\operatorname{Re}\left\{\sigma_{f}\right\}+j \operatorname{Im}\left\{\sigma_{f}\right\}=\sum_{i=0}^{n-1} r_{i} e^{-j 2 \pi f i / n}
$$

In the case of an ORM, $n$ is the total number of BPMs or correctors, while the columns of matrix $\mathbf{F}$ represent the mode space of BPMs and correctors comprised of pure sine and cosine functions. In this way, a DFT-based decomposition gives a physical interpretation to the mode space of an ORM and would be equivalent to the harmonic analysis. The inverse of $\mathbf{R}_{C}$ can be written as

$$
\mathbf{R}_{C}^{-1}=\mathbf{F}^{*} \boldsymbol{\Lambda}^{-1} \mathbf{F}
$$

where $\Lambda^{-1}$ is the diagonal matrix having inverses of Fourier coefficients at its diagonal positions. 


\section{B. Equivalence of SVD and DFT for circulant symmetry}

For the general matrices, there is no analytic information available to interpolate the SVD modes between discrete elements of $\mathbf{U}$ and $\mathbf{V}$ matrices, as SVD chooses specific mode basis vectors in order to satisfy the orthogonality of the $\mathbf{U}$ and $\mathbf{V}$ matrices. In the case of circulant matrices, there exists an equivalence between SVD and DFT by introducing the discrete Hartley transform matrix H. Following Eq. (8) and using Theorem 4.1 of Ref. [25], which states that the SVD of a circulant matrix can be written as

$$
\mathbf{R}_{C}=[\mathbf{H}(\mathbf{F}) \operatorname{Re}\{\boldsymbol{\Sigma}\}-\mathbf{H}(\overline{\mathbf{F}}) \operatorname{Im}\{\boldsymbol{\Sigma}\}]|\boldsymbol{\Lambda}| \mathbf{H}(\mathbf{F}),
$$

where

$$
\begin{aligned}
& \mathbf{H}(\mathbf{F})=\operatorname{Re}\{\mathbf{F}\}+\operatorname{Im}\{\mathbf{F}\}, \\
& \mathbf{H}(\overline{\mathbf{F}})=\operatorname{Re}\{\mathbf{F}\}-\operatorname{Im}\{\mathbf{F}\},
\end{aligned}
$$

the diagonal matrix $\boldsymbol{\Lambda}$ of the DFT-based decomposition can be written as

$$
\boldsymbol{\Lambda}=\mathbf{\Sigma}|\boldsymbol{\Lambda}|
$$

with $\boldsymbol{\Sigma}$ and $|\boldsymbol{\Lambda}|$ being the diagonal matrices containing the phases $\phi_{d i}$ and magnitudes of each Fourier coefficient, respectively. The matrices $\mathbf{U}, \mathbf{S}$, and $\mathbf{V}$ are calculated below by solving the right-hand side of Eq. (12) elementwise. The last term can be solved as

$$
\begin{aligned}
{[\mathbf{H}(\mathbf{F})]_{f, i} } & =\frac{1}{\sqrt{n}}\left[\cos \left(\frac{2 \pi f i}{n}\right)+\sin \left(\frac{2 \pi f i}{n}\right)\right] \\
& =\sqrt{\frac{2}{n}}\left[\cos \left(\frac{2 \pi f i}{n}+\phi\right)\right] \\
& =\left(\mathbf{V}^{\mathrm{T}}\right)_{f, i}
\end{aligned}
$$

where $\phi=-\frac{\pi}{4}$. The singular values of the SVD matrix $\mathbf{S}$ are the moduli of the Fourier coefficients of DFT diagonal matrix $\boldsymbol{\Lambda}$ :

$$
\mathbf{S}=|\boldsymbol{\Lambda}| .
$$

Similarly, the $\mathbf{U}$ matrix is equal to the following first part of the right-hand side of Eq. (12) as

$$
\begin{aligned}
{[\mathbf{H}(\mathbf{F}) \operatorname{Re}\{\boldsymbol{\Sigma}\}-\mathbf{H}(\overline{\mathbf{F}}) \operatorname{Im}\{\boldsymbol{\Sigma}\}]_{f, i} } \\
\quad=\sqrt{\frac{1}{n}}\left[\cos \left(\frac{2 \pi f i}{n}\right)+\sin \left(\frac{2 \pi f i}{n}\right)\right] \cos \left(\phi_{d i}\right)-\frac{1}{\sqrt{n}}\left[\cos \left(\frac{2 \pi f i}{n}\right)-\sin \left(\frac{2 \pi f i}{n}\right)\right] \sin \left(\phi_{d i}\right) \\
\quad=\sqrt{\frac{1}{n}}\left[\cos \left(\frac{2 \pi f i}{n}-\phi_{d i}\right)+\sin \left(\frac{2 \pi f i}{n}-\phi_{d i}\right)\right] \\
\quad=\sqrt{\frac{2}{n}}\left[\cos \left(\frac{2 \pi f i}{n}-\phi_{d i}-\frac{\pi}{4}\right)\right] \\
\quad=(\mathbf{U})_{f, i} .
\end{aligned}
$$

Combining Eqs. (15)-(17), Eq. (12) can be written as

$$
\mathbf{R}_{C}=\mathbf{U S V}^{\mathrm{T}},
$$

where $\mathbf{U}$ spans the space of $z$ and $\mathbf{V}$ spans the space of $\boldsymbol{\Theta}$. This conversion shows a significant difference between the two techniques in terms of information spread. SVD distributes the information in all three matrices, while DFT-based decomposition compresses all the information into one diagonal matrix. SVD-like mode truncation is also possible here by removing the Fourier coefficients of absolute values below a certain threshold along with the corresponding columns of $\mathbf{F}$, thus preserving the main benefit of SVD while adding the benefits of harmonic analysis for square circulant matrices.

\section{Broken symmetry and nearest-circulant approximation}

In many scenarios, the circulant symmetry of the ORM can be broken due to the odd placement of BPMs and correctors, the presence of insertion devices, beta beating, etc. For example, two horizontal correctors in SIS18 are placed in the second dipoles of the fourth and sixth sections, while all others are in the first dipoles, hence breaking the circulant symmetry in the corresponding columns highlighted with bold in the ORM below calculated by MAD-X for triplet optics in the units of $\mathrm{mm} / \mathrm{mrad}$ : 


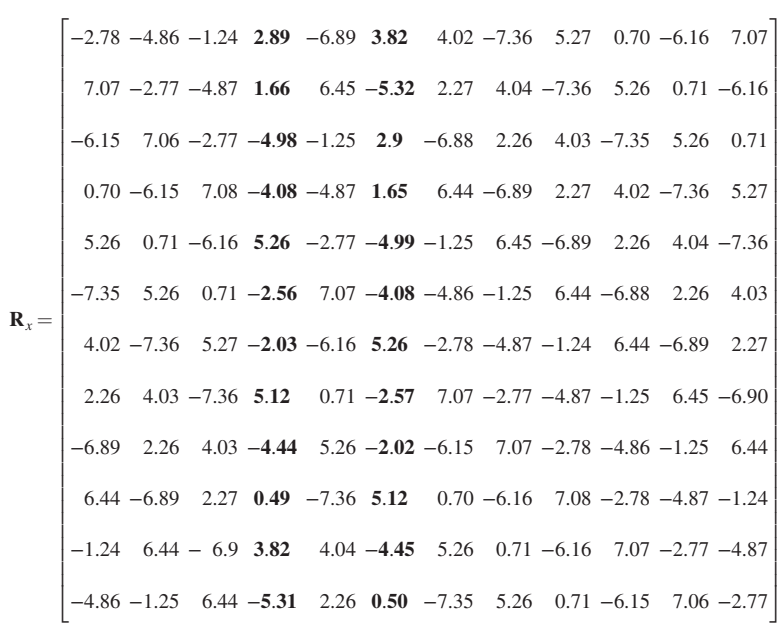

(19)

In this case, a DFT of only one row or column cannot be used directly for the decomposition and inversion of the ORM. However, a slight modification of the ORM in order to find a nearest-circulant approximation is proposed as an alternate for broken symmetries. This is based upon the fact that an iterative correction implemented for most orbit correction systems can still converge with a modified process model at the cost of more iterations or correction speed [21]. A recent example is the use of Tikhonov regularization [26] of the ORM in order to "weaken" the control action for higher-order SVD modes corresponding to smaller singular values by a replacement represented by the symbol " $\rightarrow$ " below:

$$
\frac{1}{s_{i}} \rightarrow \frac{s_{i}}{s_{i}^{2}+\mu}
$$

Here the $s_{i}$ are the singular values, and an appropriate value of $\mu>0$ serves to filter out the effect of singular values for which $s_{i}^{2} \ll \mu$, hence effectively decreasing the condition number of the ORM [27].

The theory of nearest-circulant approximation is discussed in detail in Ref. [28] but has never been explored for ORM inversion before. For a given square matrix $\mathbf{R}$, its nearest-circulant approximation $\mathbf{R}_{N C}=\operatorname{circ}\left[c_{0}, c_{1}, \ldots, c_{n-1}\right]$ can be found by the Frobenius inner product of $\mathbf{R}$ with permutation matrices $\pi_{n}^{i}$ as (see Appendix B)

$$
c_{i}=\frac{1}{n}\left\langle\mathbf{R}, \pi_{n}^{i}\right\rangle,
$$

where $n$ is the size of both $\mathbf{R}$ and $\pi$ matrices and the order of the permutation matrix is $i=0, \ldots, n-1$. Equation (21) is equivalent to the averaging of those diagonal elements of $\mathbf{R}$ for which the corresponding entries in the permutation matrix are nonzero. For the resultant approximation, the theory discussed in Sec. II A holds. Another scenario of replacing the bold columns in Eq. (19) with those that one would expect for a circulant matrix, called pure-circulant approximation, is also considered for comparison. Figure 2

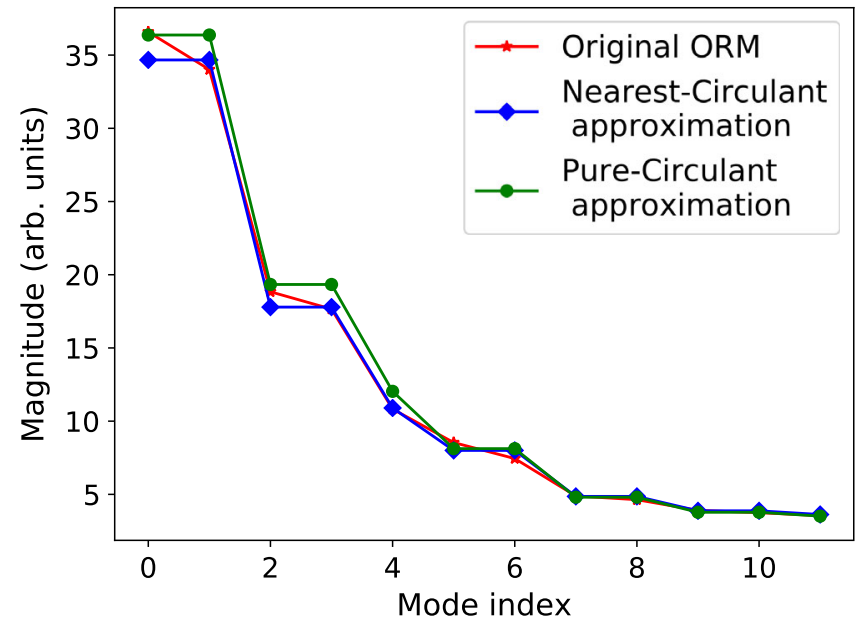

FIG. 2. Comparison of singular values of the SIS18 ORM with its nearest-circulant approximation and its pure-circulant approximation in the $x$ plane. The abscissa is the index of singular values of the matrix $\mathbf{S}$, while the ordinate is the magnitude of the singular values.

shows the singular values of the SIS18 horizontal ORM, its nearest-circulant approximation, and the pure-circulant approximation for a qualitative comparison. The effect of the nearest-circulant approximation seen as a "model deviation" on the orbit correction can be quantified in terms of the residual after one iteration of orbit correction, given as $[5,21]$

$r_{1}=z-\mathbf{R}_{M} \Theta_{N C}=z-\mathbf{R}_{M} \mathbf{R}_{N C}^{-1} z=\left(\mathbf{I}-\mathbf{R}_{M} \mathbf{R}_{N C}^{-1}\right) z$,

where $\mathbf{R}_{M}$ is the actual machine ORM and $\Theta_{N C}$ is the corrector settings vector calculated using the nearestcirculant approximation $\mathbf{R}_{N C}$ of the original model ORM R. The calculated first iteration residual (rms) for nearest- as well as pure-circulant approximations are $\simeq 13 \%$ and $\simeq 14 \%$ of the original perturbed orbit, respectively. Figure 3 shows the experimentally measured closed orbit before and after one iteration of correction in the horizontal plane of SIS18. The difference in the rms of the residual orbit is $\simeq 9 \%$ of the initial distortion for using the original ORM and its nearest-circulant approximation. It is likely that a controller which is capable of orbit correction for the original ORM will also provide the correction for its nearest-circulant approximation.

\section{Block-circulant symmetry of SIS100 ORMs}

SIS100 is the largest synchrotron of the FAIR project with a sixfold symmetry. Each of the six sections has 14 BPMs and 14 correctors, while in one section the cold quadrupole is replaced by a warm quadrupole [29]. The warm quadrupole results in a beta beating (peak-peak $\approx 10 \%$ ) and hence a loss of symmetry in the ORM as calculated by MAD-X. The beta function at BPM locations in the $y$ plane has been plotted in Fig. 4 for three 


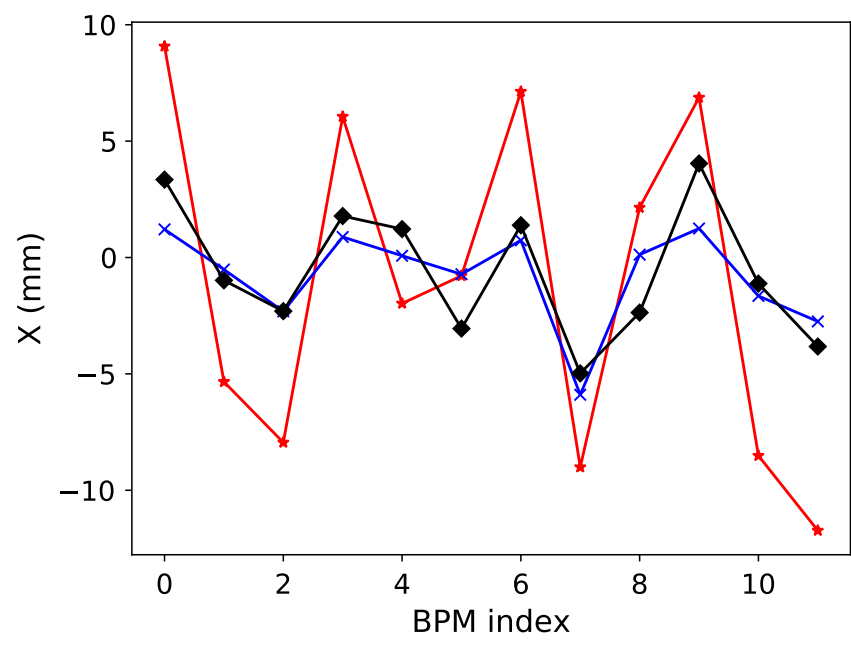

FIG. 3. Measured closed orbits in the $x$ plane of SIS18. Red line (star): Perturbed orbit $(\mathrm{rms}=7.12 \mathrm{~mm})$. Blue line (times): Corrected orbit using original ORM $(\mathrm{rms}=2.15 \mathrm{~mm})$. Black line (diamond): Corrected orbit using nearest-circulant approximation $(\mathrm{rms}=2.82 \mathrm{~mm})$.

consecutive sections with and without beta beating (by replacing the warm quadrupole by a cold quadrupole in MAD-X). The block symmetry of the SIS100 ORMs can be explored in two ways: either by (a) ignoring beta beating or by (b) finding the nearest-block-circulant approximation by averaging the diagonal blocks. In either case, the ORM attains a blockwise symmetry such that identical blocks of elements appear at the diagonal locations:

$$
\mathbf{R}_{B C}=\operatorname{circ}\left[\mathbf{A}_{0}, \mathbf{A}_{1}, \mathbf{A}_{2} \ldots \mathbf{A}_{m-1}\right] .
$$

Such a matrix is referred to as block-circulant matrix (BCM) [22]. Here $\mathbf{A}_{i}$ are the arbitrary square blocks of

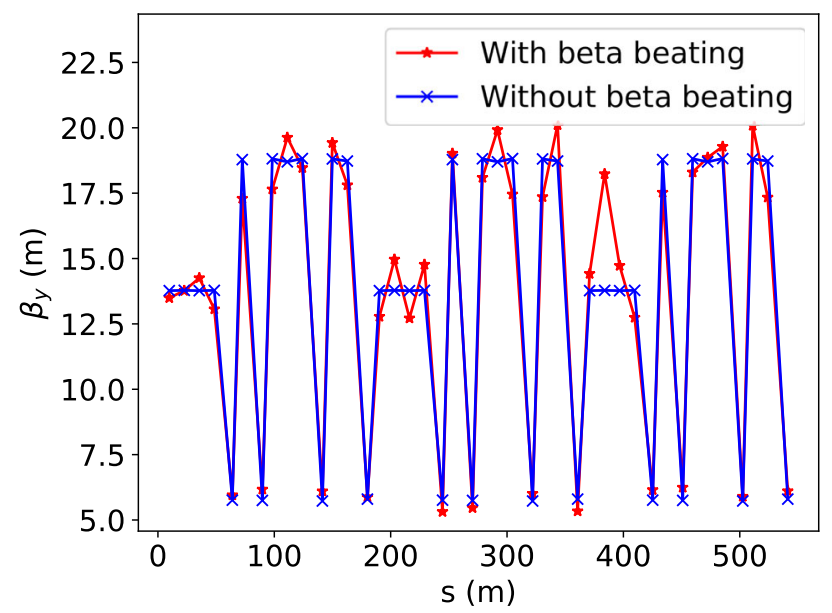

FIG. 4. Beta function at BPM locations in the $y$ plane of SIS100 (simulated in MAD-X) plotted for three cells, with beta beating [red line (star)] and without beta beating [blue line (times)]. The abscissa is the distance along the synchrotron.

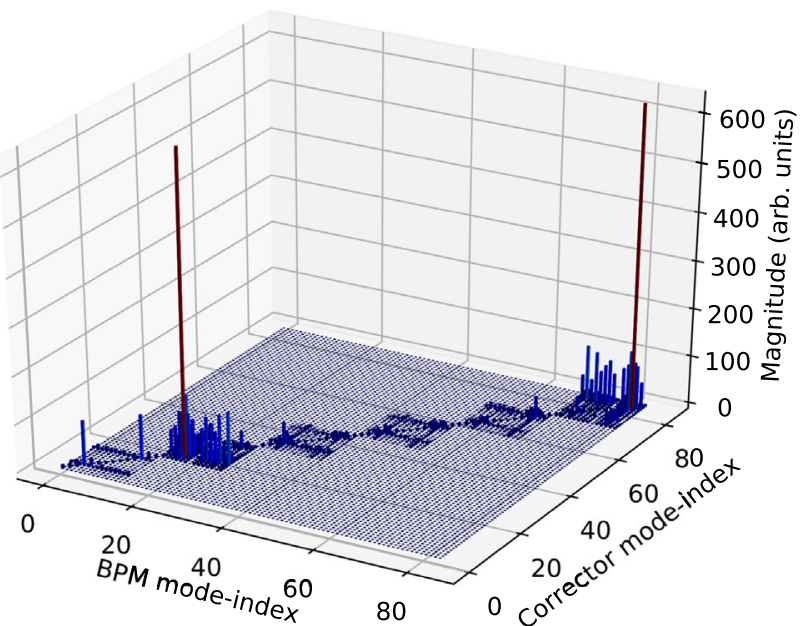

FIG. 5. Magnitudes of the complex entries of the block diagonal matrix $\mathbf{D}$ defined in Eq. (25), calculated for the case of ignoring the beta beating in the $x$ plane of SIS 100 .

dimension $n$, and $\mathbf{R}_{B C}$ is a square matrix of dimensions $m n \times m n$. For the SIS100 ORM, $m$ is the number of sections, while $n$ is the number of BPMs or correctors in each section. Here, we shall limit the discussion to the square BCM resulting from the equal number of BPMs and correctors in each section. A specific example of $n_{\mathrm{bpm}}=2 n_{\text {corrector }}$ resulting in rectangular matrices is shown in Appendix E. A square BCM can also be diagonalized with the help of Fourier matrices as [22]

$$
\mathbf{R}_{\mathrm{BC}}=\left(\mathbf{F}_{m} \otimes \mathbf{F}_{n}\right)^{*} \mathbf{D}\left(\mathbf{F}_{m} \otimes \mathbf{F}_{n}\right),
$$

where

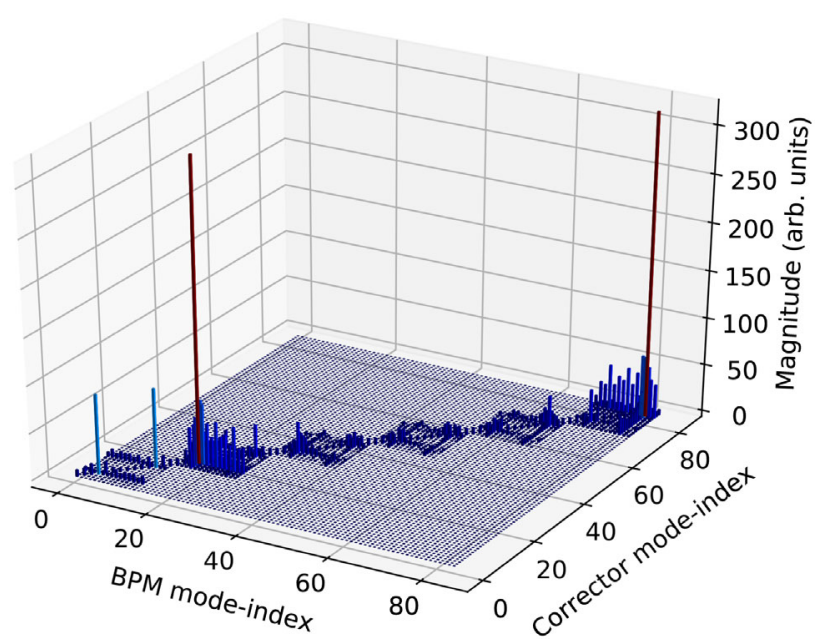

FIG. 6. Magnitudes of the complex entries of the block diagonal matrix $\mathbf{D}$ defined in Eq. (25), calculated for the case of ignoring the beta beating in the $y$ plane of SIS100. 


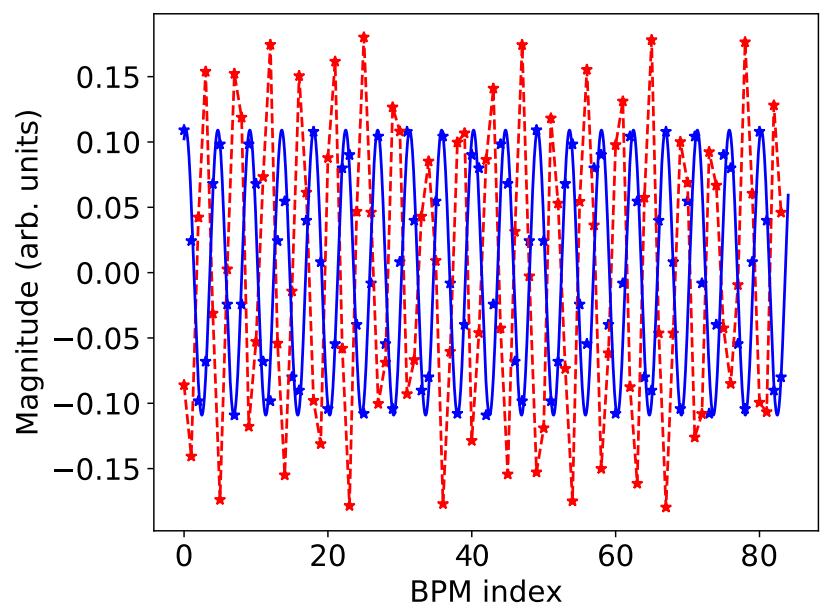

FIG. 7. Tune modes of a DFT matrix $\left(\mathbf{F}_{6}^{*} \otimes \mathbf{F}_{14}^{*}\right)$ [blue (solid) line] and SVD matrix $\mathbf{U}$ [red (dotted) line] for the SIS100 ORM in the $x$ plane. The horizontal tune is 18.87 .

$$
\mathbf{D}=\left[\begin{array}{cccccc}
\mathbf{M}_{0} & 0 & 0 & \ldots & \ldots & 0 \\
0 & \mathbf{M}_{1} & 0 & \ldots & \ldots & 0 \\
\cdots & \cdots & \ldots & \ldots & \cdots & 0 \\
\cdots & \ldots & \ldots & \ldots & \ldots & 0 \\
0 & 0 & 0 & \cdots & \cdots & \mathbf{M}_{m-1}
\end{array}\right]
$$

$\mathbf{F}_{m}$ and $\mathbf{F}_{n}$ are the standard Fourier matrices defined in Eq. (9). The symbol $\otimes$ denotes the Kronecker product of the matrices (see Appendix C). $\mathbf{M}_{i}$ are the square matrices of dimension $n$ which contain all the information of the block-circulant matrix and can be calculated using only the first row of blocks as reproduced from Ref. [22] in Appendix D. Equation (24) can be solved to calculate the inverse or pseudoinverse $\left(\mathbf{R}_{B C}^{+}\right)$of the ORM as
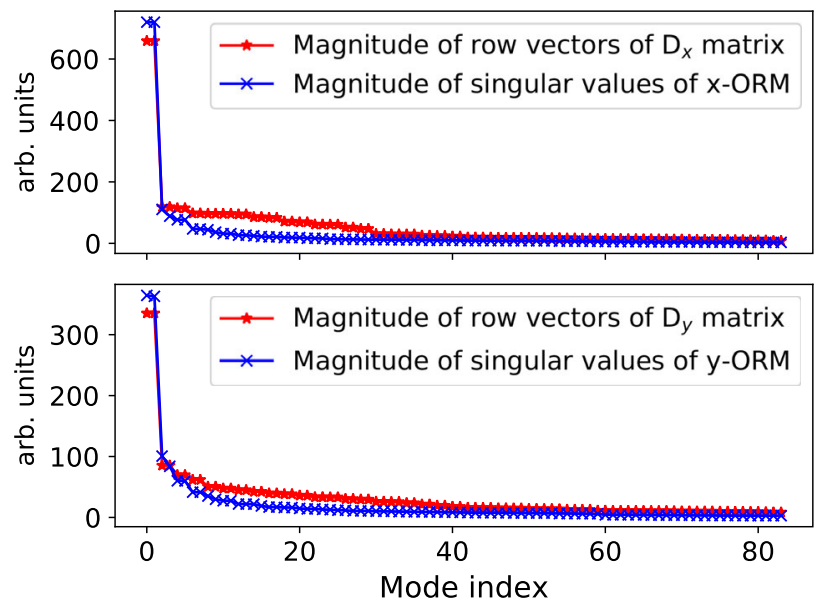

FIG. 8. Comparison of the SVD singular values with the magnitudes of the rows of matrix $\mathbf{D}$ defined in Eq. (25) for the SIS100 ORM in the $x$ plane. The abscissa is the index of singular values of the matrix $\mathbf{S}$ as well as the index of rows of matrix $\mathbf{D}$ (after sorting in descending order of their magnitude).
TABLE I. Calculated first iteration residuals in the case of symmetry exploitation in the SIS100 ORMs.

\begin{tabular}{lcc}
\hline \hline SIS100 ORM & $x$ plane & $y$ plane \\
\hline Original ORM & $0 \%$ & $0 \%$ \\
Nearest-circulant approximation & $34 \%$ & $31 \%$ \\
Nearest-block-circulant approximation & $13 \%$ & $17 \%$ \\
Ignoring beta beating & $16 \%$ & $20 \%$ \\
\hline \hline
\end{tabular}

$$
\mathbf{R}_{B C}^{+}=\left(\mathbf{F}_{m} \otimes \mathbf{F}_{n}\right)^{*}\left(\mathbf{D}^{+}\right)\left(\mathbf{F}_{m} \otimes \mathbf{F}_{n}\right),
$$

where

$$
\mathbf{D}^{+}=\operatorname{diag}\left(\mathbf{M}_{0}^{+}, \mathbf{M}_{1}^{+}, \ldots, \mathbf{M}_{m-1}^{+}\right) .
$$

The block diagonal matrices (D) of SIS100 ORMs for both the planes in the case of ignoring the beta beating are visualized in Figs. 5 and 6 as Lego plots of their absolute values. It can be seen that very few elements have larger magnitude, providing the liberty to remove weak modes before inversion just like singular values in SVD. Also, the rows of the block diagonal matrices containing the largest absolute values correspond to the tune modes of the Fourier matrices $\left(\mathbf{F}_{m} \otimes \mathbf{F}_{n}\right)$ which are plotted for both planes in Fig. 7 along with tune modes of SVD. The equal integer part of the tune in both planes explains the same location of the largest absolute values in both Lego plots. Moreover, a comparison of the singular values with the moduli of the row vectors of the block diagonal matrices in Fig. 8 provides a clear hint on the correspondence between the two methods. The deviation of the block-circulant approximations of the SIS100 ORMs from the original matrices can also be characterized using Eq. (22). The first iteration residuals $(\%)$ for the nearest-block-circulant approximation, the block-circulant approximation by ignoring the beta beating, and the nearest-circulant approximation calculated by Eq. (21) (for comparison) have been listed in Table I. One can see that the block-circulant approximation by either of the aforementioned techniques can be used for the orbit correction.

It is worth mentioning that, despite the presence of the insertion devices, the superperiodicity of the storage rings of many light sources can also be used to exploit the block-circulant symmetry in the ORM. A few examples include ALBA of Spain [30], Sirius of Brazilian Light Source [31], and NSLS2 of Brookhaven National Laboratory [32].

\section{APPLICATIONS AND DISCUSSION}

Symmetry exploitation in the ORM for DFT-based decomposition has some practical advantages over SVDbased decomposition. In this section, the benefits concerning uncertainty modeling, robustness against "missing" BPMs (e.g., ignoring a possibly faulty position reading), 
computational complexity, and removal of dispersioninduced effects from the closed orbit are demonstrated with the help of simulations and experiments for the SIS18 synchrotron of GSI.

\section{A. Uncertainty description in spatial process model}

Uncertainties appear into ORMs through various sources, e.g., BPM and corrector calibration errors and tune variation due to magnet gradient errors or during the acceleration ramp [21]. For SVD, the uncertainty $\Delta$ in the ORM appears in all three matrices of SVD which, e.g., in additive representation can be written as [20]

$$
\left(\mathbf{I}+\Delta_{R}\right) \mathbf{R}=\left(\mathbf{I}+\Delta_{U}\right) \mathbf{U}\left(\mathbf{I}+\Delta_{S}\right) \mathbf{S V}^{\mathrm{T}}\left(\mathbf{I}+\Delta_{V}\right)^{\mathrm{T}} .
$$

A previous effort reported in the literature [18], of decoupling the uncertainty from the mode space (i.e., $\mathbf{U}$ and $\mathbf{V}$ ), was to use the Fourier coefficients of harmonic analysis as a function of the betatron tune as given below:

$$
\sigma_{f}=\frac{Q_{z}}{\pi\left(Q_{z}^{2}-f^{2}\right)},
$$

where $f$ is the discrete frequency and $Q_{z}$ is the betatron tune in either transverse plane. But the lack of a quantitative relation between the Fourier coefficients expressed in Eq. (29) and the SVD singular values limits the efficacy of the method for SVD-based closed orbit correction systems. The information compression into one diagonal matrix in the case of DFT-based diagonalization, however, can provide a significant simplification of the uncertainty description as well as robust inversion of the ORM against model errors which will occur solely in the diagonal matrix, while the mode space $(\mathbf{F})$ remains fixed irrespective of any uncertainty as

$$
\begin{gathered}
\left(\mathbf{I}+\Delta_{R_{C}}\right) \mathbf{R}_{C}=\mathbf{F}^{*}\left(\mathbf{I}+\Delta_{\boldsymbol{\Lambda}}\right) \mathbf{\Lambda} \mathbf{F}, \\
\left(\mathbf{I}+\Delta_{R_{B C}}\right) \mathbf{R}_{B C}=\left(\mathbf{F}_{m}^{*} \otimes \mathbf{F}_{n}^{*}\right)\left(\mathbf{I}+\Delta_{D}\right) \mathbf{D}\left(\mathbf{F}_{m} \otimes \mathbf{F}_{n}\right) .
\end{gathered}
$$

\section{B. Orbit correction in the case of malfunctioning BPMs}

The physical interpretation of a BPM and corrector mode space provided by DFT-based decomposition of the ORM can be used to interpolate the closed orbit at the location of some malfunctioning or "missing" BPM. This is demonstrated for SIS18 in the vertical plane using MAD-X for a scenario of two consecutive BPMs being excluded. The operational scenario of BPM electronics failure due to a radiation shower happens often in hadron synchrotrons and can lead to local bumps if there is not enough redundancy in the number of BPMs.

Figure 9 shows a simulated closed orbit (red curve) perturbed as a result of random vertical misalignments (in the range of -0.85 to $0.85 \mathrm{~mm}$ ) in all 24 quadrupoles of

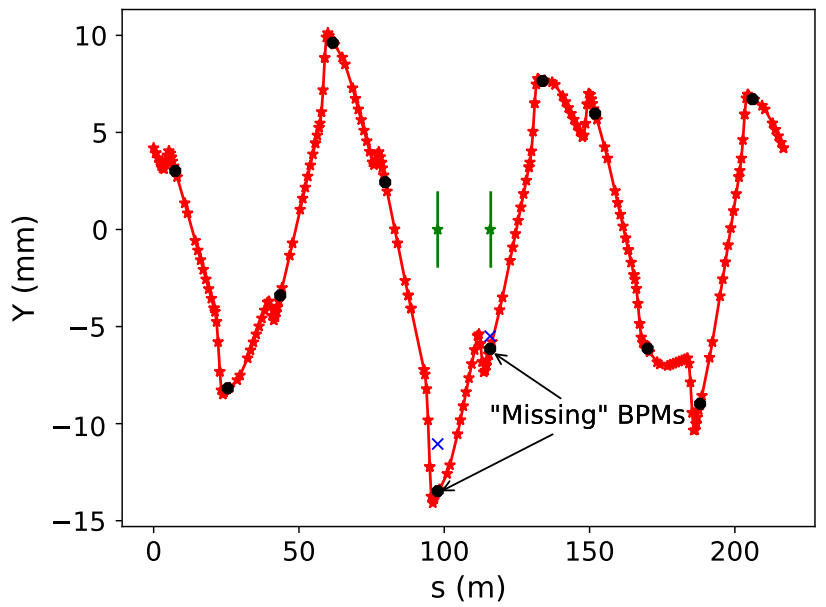

FIG. 9. Prediction of the closed orbit position at the missing BPM locations. Red (star): Simulated perturbed orbit in MAD-X in the $y$ plane of SIS18. Black (circle): Sampling of perturbed orbit at BPM locations. Green (star): Random initial guess for the orbit position at the two missing BPM locations. Blue (times): Estimated orbit position at missing BPM locations using the DFT mode structure of the ORM.

SIS18. A misaligned quadrupole has the effect of a magnetic dipole on the beam, and as a result the closed orbit is perturbed from its "ideal path." The black dots in Fig. 9 represent the sampling of the perturbed orbit at the locations of BPMs, while the green dots show the mean values of random orbit positions (in a range of -1 to $1 \mathrm{~mm}$ marked as error bars around the green dots) assumed at the location of missing or faulty BPMs. A combination of cosine functions with discrete frequencies $f=3,4,2,5$ (corresponding to dominant Fourier modes) was used to estimate the orbit position at the missing BPM locations, keeping their relative amplitudes and phases as free parameters to be optimized. The fitting algorithm was constrained to keep the fitted curve closest to the orbit positions within an accuracy of $0.01 \mathrm{~mm}$ at the working BPM locations while free to choose any value at the location of missing BPMs. As a result, the optimized orbit positions are found to be closer to the actual orbit positions within maximum errors of $3 \pm 0.048$ and $0.5 \pm 0.048 \mathrm{~mm}$, respectively. Figure 10 shows the simulated orbit correction using the estimated orbit positions (green curve). The red curve shows the perturbed orbit without corrections, while the magenta curve shows the corrected orbit when all BPMs are working. The orbit correction using SVD of the noncirculant matrix (excluding the rows in the ORM corresponding to the faulty BPMs) is plotted in black. The orbit correction taking the orbit position "zero" at the missing BPM locations and using a circulant matrix is also plotted as a blue curve for comparison. The robustness against missing BPMs is shown by the overall improved correction obtained using an estimated beam position instead of using the noncirculant matrix. Besides the better global correction, one can also get the benefits of circulant 


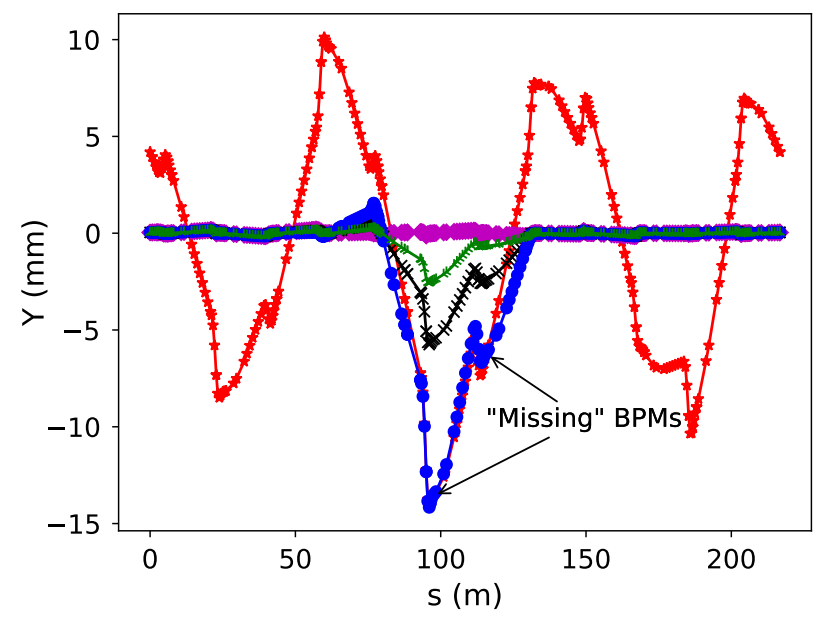

FIG. 10. Simulation of orbit correction for various cases of missing BPMs. Red (star): Simulated perturbed orbit in MAD-X in the $y$ plane of SIS18. Magenta (diamond): Corrected orbit using all BPMs. Green (tri_up): Corrected orbit using the predicted orbit position for the missing BPMs. Black (times): Corrected orbit excluding the rows corresponding to the missing BPMs from the ORM. Blue (circle): Corrected orbit by taking the zero orbit position at the missing BPM locations.

symmetry and DFT-based decomposition (e.g., online decomposition during ramp) even when the symmetry has been broken due to the missing BPMs.

\section{Computational complexity}

DFT-based diagonalization and inversion of the ORM has a significant computational benefit over SVD. For a square matrix of dimension $n$, the numerical complexities for the SVD of the whole matrix and the DFT of its first row are $\mathcal{O}\left(4 n^{3}\right)$ [33] and $\mathcal{O}\left(n^{2}\right)$ [34], respectively. Such a reduction in numerical complexity becomes meaningful for larger ORMs and for synchrotrons having a continuously changing ORM within a cycle and also from one cycle to another. SIS18 is an example [21] of the latter, where lattice settings as well as the ORM change systematically during the ramp. In such cases, the possibility of online DFT-based ORM inversion becomes attractive from the perspective of computational speed as well as storage and transfer of the inverted ORMs.

\section{Momentum mismatch and orbit correction}

A mismatch between the rf frequency and the dipole field results in a relative mismatch in the average momentum of the beam $\frac{\Delta p}{p}$ and a closed orbit deviation from the equilibrium position primarily in the $x$ plane (in the absence of coupling) [35]:

$$
\Delta x_{D}(s)=D_{x}(s) \frac{\Delta p}{p} .
$$

Here $D_{x}(s)$ is the dispersion function, and $\Delta x_{D}(s)$ is the resultant shift in the closed orbit. Figure 11 shows a set of

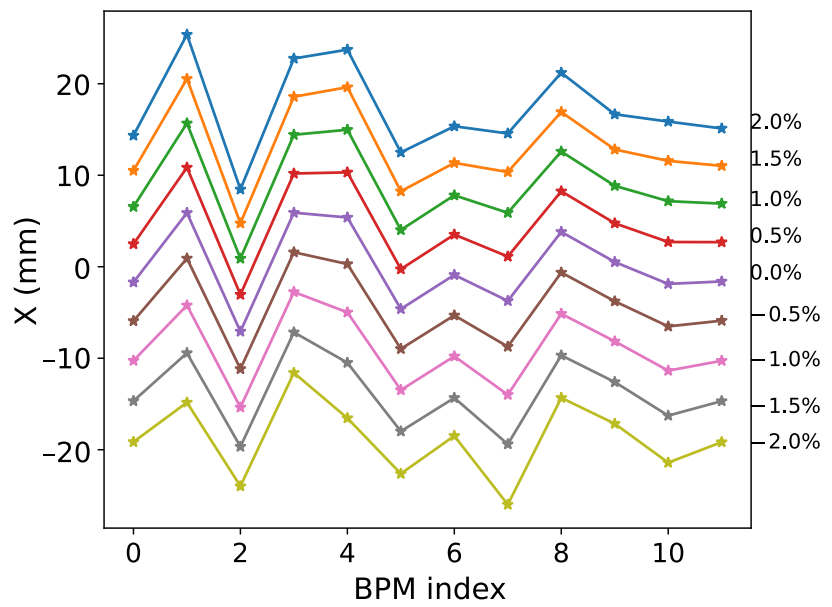

FIG. 11. Measured horizontal closed orbits in SIS18 for the various average momentum offsets represented as percent with each orbit.

measured horizontal dispersion orbits for a relative momentum mismatch in the range of $-2 \%$ to $2 \%$ with a step size of $0.5 \%$ in SIS18 at the extraction energy. One can see a shift of the mean value of the closed orbit as a function of $\frac{\Delta p}{p}$. An attempt to correct such an orbit shift can saturate the corrector magnets. Therefore, the contribution from dispersion needs to be subtracted from the closed orbit before the correction. Generally, the dispersion-induced orbit distortion will couple into many modes of BPM space, depending on the sampling of the dispersion function.

For SIS18, the dispersion function has the same value $D(s)=D_{0}$ at all the BPM locations, and the resultant dispersion-induced dc part of the closed orbit will couple to the pure dc mode of the BPM space corresponding to $f=0$ in the case of a circulant ORM and can be ignored by removing the singular value corresponding to that mode (the last column of $\mathbf{U}$ ). Figure 12 shows the coupling of the
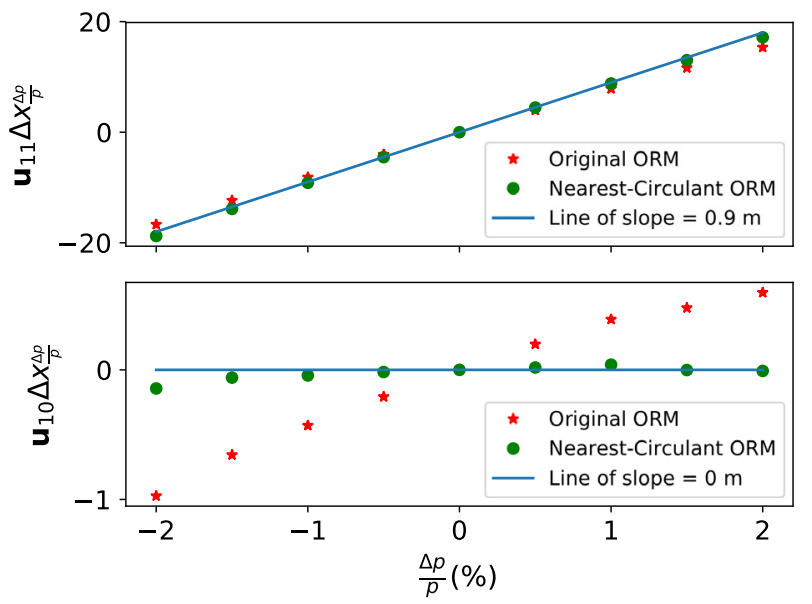

FIG. 12. Coupling of experimentally measured dc shift $\Delta x$ $(\mathrm{mm})$ in the horizontal closed orbit to the last two columns of the $\mathbf{U}$ matrices $\left(\mathbf{u}_{11}\right.$ and $\mathbf{u}_{10}$ ) of the original ORM and its nearestcirculant approximation. 
dc part of the measured closed orbit to the last two modes of the original noncirculant ORM and its nearest-circulant approximation for SIS18. It is evident that the dc shift of the closed orbit couples to the last mode of the matrix $\mathbf{U}$, as $\mathbf{u}_{11} \Delta x_{\frac{\Delta p}{p}}$ increases linearly with $\frac{\Delta p}{p}$ with a slope of $0.9 \mathrm{~m}$ (equal to the value of dispersion function $D_{0}$ at the BPM locations). The slight deviation from this behavior in the case of the original ORM is due to a slight distortion of the last mode from pure dc structure due to the broken symmetry. This results in the coupling of the dc part of the closed orbit to higher modes as well, as is evident in Fig. 12. Here in the case of nearest-circulant approximation there is almost zero coupling to the second-last mode. This shows the benefit of circulant symmetry in the ORMs in order to reject energy dispersion in the closed orbit by simply discarding the last dc mode of the matrix $\mathbf{U}$, instead of measuring and subtracting it from the closed orbit before the correction. The projection of dispersion into the last mode of $\mathbf{U}$ can also be utilized to synchronize the $\mathrm{rf}$ frequency and the dipole field.

\section{CONCLUSION}

An efficient method relying on circulant symmetry properties in synchrotrons for the diagonalization and inversion of the ORM is introduced in this paper. A circulant ORM can be decomposed into BPM and corrector vector spaces with the help of a one-dimensional DFT; this method is significantly faster than the SVD technique and provides a physical interpretation of the mode space. A relation between DFT coefficients and SVD singular values as well as modes is discussed which shows that DFT-based decomposition contains the benefits of harmonic analysis and SVD. Furthermore, the block-circulant symmetry arising from the blockwise periodicity of a lattice is shown to have SVD-like robustness benefits in line with the purecirculant symmetry case under DFT decomposition. These results are extended to broken symmetries with the nearestcirculant approximation, hence making it applicable to a wider range of magnet lattices. DFT-based decomposition provides information compression into the diagonal matrix only, which can significantly simplify uncertainty modeling in the ORM, overcoming a deficiency of SVD decomposition where the information is distributed among all three matrices. The usefulness of the transparent interpretation of the BPM and corrector spaces as Fourier modes is demonstrated for the SIS18 synchrotron at FAIR by interpolating the closed orbit to the location of missing BPMs to achieve a better global orbit correction. The reduced computational complexity of DFT can have benefits concerning the overall performance of closed orbit feedback systems in the case of large ORMs and during the energyramp orbit correction. A new technique for the rejection of the dispersion-induced orbit shift during orbit correction is introduced and verified with measurements at SIS18.
The methods are presented in the context of the FAIR synchrotrons but would apply to any synchrotron or storage rings with the aforementioned symmetry or a slightly broken symmetry. Thus, we emphasize the importance of symmetry exploitation in the ORM and consideration at the design stage of the closed orbit feedback system.

\section{ACKNOWLEDGMENTS}

The authors thank Thomas Reichert and Will Stem for reading the paper, and S. H. M. expresses gratitude to Guenther Rehm from Diamond Light Source United Kingdom, for useful discussions, and to Daniel O. Tavares from Brazilian Light Source, for providing useful information about symmetric ORMs in light sources. This project has received funding from the European Unions Horizon 2020 Research and Innovation program under Grant Agreement No. 730871 (ARIES). S. H. M. thankfully acknowledges the financial support from DAAD (Deutscher Akademischer Austauschdienst/German Academic Exchange Service) for his doctoral research work under Personal Reference No. 91605207.

\section{APPENDIX A: PERMUTATION MATRIX}

A general permutation matrix $\pi_{n}^{i}$ is a square matrix of size $n$ which can be generated as a result of the cyclic permutations of the identity matrix. The number of permutations $i(0 \leq i \leq n-1)$ is called the order of the permutation matrix. The identity matrix is a permutation matrix of the order of 0 . The set of fundamental permutation matrices for a dimension of 3 are shown below:

$$
\begin{gathered}
\pi_{3}^{0}=\left[\begin{array}{lll}
1 & 0 & 0 \\
0 & 1 & 0 \\
0 & 0 & 1
\end{array}\right], \quad \pi_{3}^{1}=\left[\begin{array}{lll}
0 & 1 & 0 \\
0 & 0 & 1 \\
1 & 0 & 0
\end{array}\right], \\
\pi_{3}^{2}=\left[\begin{array}{lll}
0 & 0 & 1 \\
1 & 0 & 0 \\
0 & 1 & 0
\end{array}\right] .
\end{gathered}
$$

\section{APPENDIX B: FROBENIUS INNER PRODUCT}

The symbol \langle\rangle used in Eq. (21) denotes the Frobenius inner product, which is a binary operation that takes two matrices of equal size as input and returns a number as a result of adding the elementwise inner products. For two real $m \times n$ matrices $\mathbf{A}$ and $\mathbf{B}$, it is defined as

$$
\langle\mathbf{A}, \mathbf{B}\rangle=\sum_{i=1}^{m} \sum_{k=1}^{n} a_{i k} b_{i k} .
$$




\section{APPENDIX C: KRONECKER PRODUCT OF MATRICES}

Given an $m \times n$ matrix $\mathbf{A}$ and $p \times q$ matrix $\mathbf{B}$, the Kronecker product $\mathbf{C}=\mathbf{A} \otimes \mathbf{B}$ is a matrix of size $m p \times n q$ given as

$$
\mathbf{A} \otimes \mathbf{B}=\left[\begin{array}{ccccc}
a_{11} \mathbf{B} & \ldots & \ldots & \ldots & a_{1 n} \mathbf{B} \\
\ldots & \ldots & \ldots & \ldots & \ldots \\
\cdots & \ldots & \ldots & \ldots & \ldots \\
\ldots & \ldots & \ldots & \ldots & \ldots \\
a_{m 1} \mathbf{B} & \ldots & \ldots & \ldots & a_{m n} \mathbf{B}
\end{array}\right]
$$

\section{APPENDIX D: DIAGONALIZATION OF BCMS}

This Appendix is adopted from Ref. [22] (Theorem 5.6.4), where a BCM can also be written with the help of fundamental permutation matrices by replacing the numbers with matrices $\mathbf{A}_{i}$ in Eq. (6) as

$$
\mathbf{R}_{B C}=\sum_{i=0}^{n-1}\left(\pi_{m}^{i} \otimes \mathbf{A}_{i}\right)
$$

For arbitrary $\mathbf{A}_{i}$,

$$
\pi_{m}^{i} \otimes \mathbf{A}_{i}=\left(\mathbf{F}_{m}^{*} \Omega^{i} \mathbf{F}_{m}\right) \otimes \mathbf{F}_{n}^{*}\left(\mathbf{F}_{n} \mathbf{A}_{i} \mathbf{F}_{n}^{*}\right) \mathbf{F}_{n}
$$

Let $\mathbf{B}_{i}=\mathbf{F}_{n} \mathbf{A}_{i} \mathbf{F}_{n}^{*}$, and then Eq. (D1) becomes

$$
\mathbf{R}_{B C}=\left(\mathbf{F}_{m} \otimes \mathbf{F}_{n}\right)^{*}\left(\sum_{i=0}^{n-1} \Omega^{i} \otimes \mathbf{B}_{i}\right)\left(\mathbf{F}_{m} \otimes \mathbf{F}_{n}\right) .
$$

The middle term in Eq. (D3) has a form of a diagonal matrix as

$$
\sum_{i=0}^{n-1} \Omega^{i} \otimes \mathbf{B}_{i}=\operatorname{diag}\left(\mathbf{M}_{0}, \mathbf{M}_{1}, \ldots, \mathbf{M}_{m-1}\right)=\mathbf{D},
$$

where $\mathbf{M}_{i}$ can be calculated by the relation

$$
\left[\begin{array}{c}
\mathbf{M}_{0} \\
\mathbf{M}_{1} \\
\cdots \\
\cdots \\
\mathbf{M}_{m-1}
\end{array}\right]=\left(m^{1 / 2} \mathbf{F}_{m}^{*} \otimes \mathbf{I}_{n}\right)\left[\begin{array}{c}
\mathbf{B}_{0} \\
\mathbf{B}_{1} \\
\cdots \\
\cdots \\
\mathbf{B}_{m-1}
\end{array}\right]
$$

and Eq. (D3) becomes

$$
\mathbf{R}_{B C}=\left(\mathbf{F}_{m} \otimes \mathbf{F}_{n}\right)^{*}(\mathbf{D})\left(\mathbf{F}_{m} \otimes \mathbf{F}_{n}\right) .
$$

\section{APPENDIX E: BLOCK-CIRCULANT SYMMETRY IN THE CASE OF $\boldsymbol{n}_{\mathrm{bpm}}=\mathbf{2} \boldsymbol{n}_{\text {corrector }}$}

The concept of DFT-based decomposition of the ORM can also be extended to the matrices where $n_{\mathrm{bpm}}=2 n_{\text {corrector }}$, and the orbit response matrix can be arranged in the form of two circulant blocks each of dimension $n$ as

$$
\mathbf{R}=\left[\begin{array}{ccccccc}
a_{0} & a_{1} & a_{2} & a_{3} & \cdots & \cdots & a_{n-1} \\
a_{n-1} & a_{0} & a_{1} & a_{2} & \cdots & \cdots & a_{n-2} \\
\cdots & \cdots & \cdots & \cdots & \cdots & \cdots & \cdots \\
a_{1} & a_{2} & a_{3} & a_{4} & \cdots & \cdots & a_{0} \\
b_{0} & b_{1} & b_{2} & b_{3} & \cdots & \cdots & b_{n-1} \\
b_{n-1} & b_{0} & b_{1} & b_{2} & \cdots & \cdots & b_{n-2} \\
\cdots & \cdots & \cdots & \cdots & \cdots & \cdots & \cdots \\
b_{1} & b_{2} & b_{3} & b_{4} & \cdots & \cdots & b_{0}
\end{array}\right]
$$

and can be diagonalized by the DFT of the first rows of each block as

$$
\mathbf{R}=\left(\frac{1}{n}\right) \mathbf{F}_{c}^{*} \boldsymbol{\Lambda}_{c} \mathbf{F}
$$

where $\mathbf{F}$ is the standard Fourier matrix of size $n . \mathbf{F}_{c}$ is a $2 n \times 2 n$ matrix constructed using the standard Fourier matrix of size $n$ as

$$
\mathbf{F}_{c}=\left[\begin{array}{cc}
\mathbf{F} & \mathbf{0} \\
\mathbf{0} & \mathbf{F}
\end{array}\right]
$$

and $\boldsymbol{\Lambda}_{c}$ is a rectangular matrix composed of two diagonal block matrices as

$$
\boldsymbol{\Lambda}_{c}=\left[\begin{array}{ccccccc}
\sigma_{a, 0} & 0 & 0 & 0 & \cdots & \cdots & 0 \\
0 & \sigma_{a, 1} & 0 & 0 & \cdots & \cdots & 0 \\
\cdots & \cdots & \cdots & \cdots & \cdots & \cdots & \cdots \\
0 & 0 & 0 & 0 & \cdots & \cdots & \sigma_{a, n-1} \\
\sigma_{b, 0} & 0 & 0 & 0 & \cdots & \cdots & 0 \\
0 & \sigma_{b, 1} & 0 & 0 & \cdots & \cdots & 0 \\
\cdots & \cdots & \cdots & \cdots & \cdots & \cdots & \cdots \\
0 & 0 & 0 & 0 & \cdots & \cdots & \sigma_{b, n-1}
\end{array}\right]
$$

where $\sigma_{a, i}$ and $\sigma_{b, i}$ are the Fourier coefficients of the first rows of each block of $\mathbf{R}$, respectively. The final decomposition can be written as

$$
\mathbf{R}=\left[\begin{array}{cc}
\mathbf{F}^{*} & \mathbf{0} \\
\mathbf{0} & \mathbf{F}^{*}
\end{array}\right]\left[\begin{array}{c}
\boldsymbol{\Lambda}_{a} \\
\boldsymbol{\Lambda}_{b}
\end{array}\right][\mathbf{F}],
$$

and the pseudoinverse of $\mathbf{R}$ can be calculated as 


$$
\mathbf{R}^{+}=\left[\mathbf{F}^{*}\right]\left[\begin{array}{ll}
\boldsymbol{\Lambda}_{a}^{+} & \boldsymbol{\Lambda}_{b}^{+}
\end{array}\right]\left[\begin{array}{cc}
\mathbf{F} & \mathbf{0} \\
\mathbf{0} & \mathbf{F}
\end{array}\right]
$$

Moreover, the SVD singular values of the matrix $\mathbf{R}$ also have a direct relation to the Fourier coefficients of individual blocks as

$$
s_{i}=\sqrt{\left|\sigma_{a, i}\right|^{2}+\left|\sigma_{b, i}\right|^{2}}
$$

[1] G. Rehm, Achieving and measuring sub-micrometer beam stability at 3rd generation light sources, J. Phys. Conf. Ser. 425, 042001 (2013).

[2] G. Decker, Beam stability in synchrotron light sources, in Proceedings of the 7th European Workshop on Beam Diagnostic and Instrumentation for Particle Accelerators (DIPAC 2005), Lyon, France, 2005 (JACoW, Geneva, 2005), p. 233.

[3] R. J. Steinhagen, LHC orbit feedback tests at the SPS, in Proceedings of the 9th European Particle Accelerator Conference (EPAC 2004), Lucerne, 2004 (EPS-AG, Lucerne, 2004), p. 2499.

[4] M. Sands, The physics of electron storage rings: An introduction, Stanford Linear Accelerator Center Report No. SLAC-121, 1970.

[5] Y. Chung, G. Decker, and K. Evans, Closed orbit correction using singular value decomposition of the response matrix, Argonne National Laboratory, 1993 (unpublished).

[6] V. Ziemann, Response matrices in strongly coupled storage rings with a radio-frequency system constraining the revolution time, Phys. Rev. ST Accel. Beams 18, 054001 (2015).

[7] A. Wolski, Introduction to Beam Dynamics in HighEnergy Electron Storage Rings (Morgan \& Claypool, San Rafael, CA, 2018), Chap. 2.7.

[8] S. Sorge, Simulation study on beam loss in the alpha bucket regime during SIS-100 proton operation, Nucl. Instrum. Methods Phys. Res., Sect. A 882, 129 (2018).

[9] Y. Cheng, Dynamic closed orbit correction, in Proceedings of the 15th Particle Accelerator Conference, PAC-1993, Washington, DC, 1993 (IEEE, New York, 1993), p. 2269.

[10] B. Autin and Y. Marti, Closed orbit correction of alternating gradient machines using a small number of magnets, European Organization for Nuclear Research Report No. CERN-ISR-MA/73-17, 1973.

[11] L. H. Yu, E. Bozoki, J. Galayda, S. Krinsky, and G. Vignola, Real time harmonic closed orbit correction, Nucl. Instrum. Methods Phys. Res., Sect. A 284, 268 (1989).

[12] V. Ziemann, Corrector ironing in the SLC final focus, in Proceedings of the 15th Particle Accelerator Conference, PAC-1993, Washington, DC, 1993 (IEEE, New York, 1993), p. 498.

[13] K. Harada, T. Obina, Y. Kobayashi, N. Nakamura, H. Takaki, and H. Sakai, Orbit correction using an eigenvector method with constraints for synchrotron radiation sources, Nucl. Instrum. Methods Phys. Res., Sect. A 604, 481 (2009).
[14] A. Friedman and E. Bozoki, Use of eigenvectors in understanding and correcting storage ring orbits, Nucl. Instrum. Methods Phys. Res., Sect. A 344, 269 (1994).

[15] S. G. Mallat and Z. Zhang, Matching pursuits with timefrequency dictionaries, IEEE Trans. Signal Process. 41, 3397 (1993).

[16] J. H. Friedman and W. Stuetzle, Projection pursuit regression, J. Am. Stat. Assoc. 76, 817 (1981).

[17] G. H. Golub and C. F. V. Loan, Matrix Computations (Johns Hopkins University, Baltimore, 2013), Chap. 2.

[18] S. Gayadeen, Uncertainty modelling of response matrix, in Proceedings of the 15th International Conference on Accelerators and Large Experimental Physics Control Systems (ICALEPCS 2015), Melbourne, Australia, 2015 (JACoW, Geneva, 2015), p. 506.

[19] S. H. Mirza, P. Forck, H. Klingbeil, and R. Singh, Investigations of spatial process model for the closed orbit feedback system at the SIS18 synchrotron at GSI, in Proceedings of the 16th International Conference on Accelerators and Large Experimental Physics Control Systems (ICALEPCS 2017), Barcelona, Spain, 2017 (JACoW, Geneva, 2017), p. 1301.

[20] S. Gayadeen, Synchrotron electron beam control, Ph.D. thesis, Department of Engineering Science, University of Oxford, United Kingdom, 2014, https://ora.ox.ac.uk/ objects/uuid:234025b3-2d1b-495e-846e-688f14149b21.

[21] S. H. Mirza, P. Forck, H. Klingbeil, and R. Singh, Effect of model errors on the closed orbit correction at the SIS18 synchrotron of GSI, J. Phys. Conf. Ser. 1067, 072035 (2018).

[22] P. J. Davis, Circulant Matrices (AMS Chelsea, Providence, 1994).

[23] H. Grote, F. Schmidt, L. Deniau, and G. Roy, Computer code MAD-X: Users reference manual, European Organization for Nuclear Research, 2016, http://mad.web.cern.ch/ $\mathrm{mad} / \mathrm{releases} / 5.02 .08 / \mathrm{madxuguide.pdf}$.

[24] K. R. Rao and P. C. Yip, The Transform and Data Compression Handbook (CRC, Boca Raton, FL, 2000).

[25] H. Karner, J. Schneid, and C. W. Ueberhuber, Spectral decomposition of real circulant matrices, Linear Algebra Appl. 367, 301 (2003).

[26] A. Neumaier, Solving ill-conditioned and singular linear systems: A tutorial on regularization, SIAM Rev. 40, 636 (1998).

[27] A. Edelman, Eigenvalues and condition numbers of random matrices, Ph.D. thesis, Mathematics Department, MIT United Kingdom, 1989, http://math.mit.edu/ edelman/ publications/eigenvalues_and_condition_numbers.pdf.

[28] M. T. Chu and R. J. Plemmons, Real-valued, low rank, circulant approximation, SIAM J. Matrix Anal. Appl. 24, 645 (2003).

[29] FAIR baseline technical report, GSI, 2006.

[30] M. Munoz and D. Einfeld, Lattice and beam dynamics of the ALBA storage ring, ICFA Beam Dyn. Newslett. 44, 183 (2007).

[31] L. Liu, F. H. de Sa, and X. R. Resende, A new optics for SIRIUS, in Proceedings of the 7th International Particle Accelerator Conference (IPAC 2016), Busan, Korea, 2016 (JACoW, Geneva, 2016), p. 3413.

[32] Y. Tian, K. Ha, L. Yu, W. Cheng, J. DeLong, and L. Dalesio, NSLS-II fast orbit feedback system, in 
Proceedings of the 15th International Conference on Accelerators and Large Experimental Physics Control Systems (ICALEPCS 2015), Melbourne, Australia, 2015 (JACoW, Geneva, 2015), p. 1.

[33] A. Frieze, R. Kannan, and S. Vempala, Fast Monte-Carlo algorithms for finding low-rank approximations, J. Assoc. Comput. Mach. 51, 1025 (2004).
[34] W. H. Press, S. A. Teukolsky, W. T. Vetterling, and B. P. Flannery, Numerical Recipes: The Art of Scientific Computing (Cambridge University Press, Cambridge, England, 2007), p. 609.

[35] K. Wille, The Physics of Particle Accelerators: An Introduction (Oxford University, New York, 2000). 